C-A/AP/\#255

October 2006

\title{
RHIC Beam-Based Sextupole Polarity Verification
}

\author{
Y. Luo, T. Satogata, P. Cameron, A. DellaPenna, D. Trbojevic
}

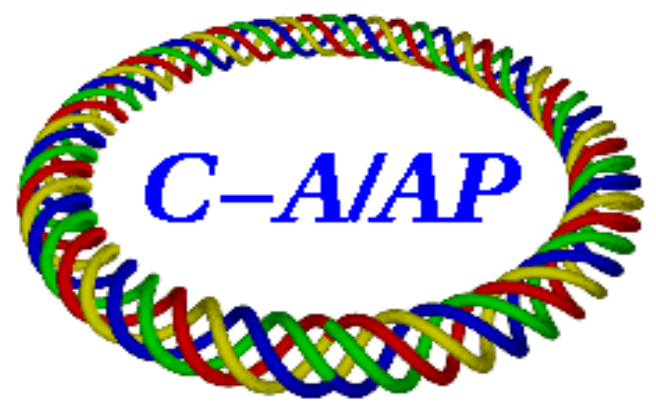

Collider-Accelerator Department Brookhaven National Laboratory Upton, NY 11973 
$\mathrm{CAD} / \mathrm{AP} / 255$

October 2, 2006

\title{
RHIC Beam-Based Sextupole Polarity Verification
}

\author{
Y. Luo, T. Satogata, P. Cameron, A. Dellapenna, and D. Trbojevic \\ Brookhaven National Laboratory, Upton, NY 11973, USA
}

This note presents a beam-based method to check RHIC arc sextupole polarities using local horizontal orbit three-bumps at injection energy. We use 11 bumps in each arc, each covering two SFs (focusing sextupoles) and one SD (defocusing sextupole). If there are no wrong sextupole polarities, the tune shifts from bump to bump and the tune shift patterns from arc to arc should be similar. Wrong sextupole polarities can be easily identified from mismatched signs or amplitudes of tune shifts from bump to bump and/or from arc to arc. Tune shifts in both planes during this study were tracked with a high-resolution baseband tunemeter (BBQ) system. This method was successfully used to the sextupole polarity check in RHIC Blue and Yellow rings in the RHIC 2006 run.

\section{Principle}

\subsection{General solution: sextupole based method}

In the simple case where a single sextupole is covered by a local horizontal orbit bump, the horizontal and vertical tune shifts are:

$$
\left\{\begin{array}{rl}
\Delta Q_{x} & =\frac{1}{4 \pi} \beta_{x}\left(k_{2} L\right) \Delta x_{c o}, \\
\Delta Q_{y} & =-\frac{1}{4 \pi} \beta_{y}\left(k_{2} L\right) \Delta x_{c o}
\end{array},\right.
$$

where $\left(\beta_{x}, \beta_{y}\right)$ are the horizontal and vertical beta functions at the sextupole, $\left(k_{2} L\right)$ is the integrated sextupole strength, and $x_{c o}$ is the horizontal orbit change due to the bump. If $x_{c o}$ is positive in Eq. 1, the sign of $\left(k_{2} L\right)$, or the sextupole polarity, is only decided by the signs of the tune shifts. For simplicity, in the following, we call a sextupole with positive polarity $k_{2}>0$ an SF sextupole, and call a sextupole with negative polarity $k_{2}<0$ an SD sextupole. The most general method is only useful when there is a single sextupole in the horizontal three-bump.

\section{$1.2 \quad$ RHIC sextupole locations}

Each ring of RHIC consists of six arcs and 144 sextupoles. Each arc has 11 periodic FODO cells. There are $12 \mathrm{SFs}$ and $12 \mathrm{SDs}$ in each arc. The phase advance per FODO cell is about $85^{\circ}$ in each plane. There is one SF sextupole and one horizontal dipole corrector close to each arc focusing quadrupole, and one SD sextupole and one vertical dipole corrector close to each arc defocusing quadrupole in the regular arc FODO lattice. There are no horizontal dipole correctors close to defocusing quadrupoles, or vertical dipole correctors close to focusing quadrupoles. One defocusing SD sextupole in each arc is located outside of the standard FODO cell arc. 144 local three-bumps are required to cover all 144 sextupoles in each RHIC ring.

As shown in Fig. 1, it is not possible to create a horizontal orbit three-bump to only cover one sextupole in the RHIC arcs. These individual sextupole-based local horizontal three bumps always cover two to four sextupoles. The tune shifts are therefore different for these bumps even if the closed orbit bumps at the sextupoles are the same size. This complication makes it difficult to identify individual sextupole polarity problems by simply looking at the tune shift patterns during data acquisition.

\subsection{Solution with multiple sextupoles per bump}

Fig. 2 shows 13 three bumps constructed with horizontal correctors in the RHIC blue ring 6-7 o'clock arcs. Since each horizontal corrector is located near a FODO lattice beta max and SF sextupole, the center 11 horizontal three-bumps change the orbit significantly at one SF and two SD sextupoles, while the end two 
horizontal three-bumps change the orbit significantly at only one SD, or one SD and one SF sextupole. A single sextupole polarity reversal can then be found by an anomalous tune shift from two adjacent bumps; the magnitude and direction of the anomaly discriminates between SF or SD sextupole reversals. The end bumps are not in the periodic RHIC arc FODO lattice, so their setup is slightly different than the center 11 horizontal three-bumps.

We set the kicking strengths for the first dipole correctors of each bumps same. The strengths for the following other two dipole correctors are given by

$$
\left\{\begin{array}{l}
\frac{\theta_{2}}{\theta_{1}}=-\sqrt{\frac{\beta_{1}}{\beta_{2}}} \frac{\sin \left(\phi_{3}-\phi_{1}\right)}{\sin \left(\phi_{3}-\phi_{2}\right)} \\
\frac{\theta_{3}}{\theta_{1}}=-\sqrt{\frac{\beta_{1}}{\beta_{3}}} \frac{\sin \left(\phi_{2}-\phi_{1}\right)}{\sin \left(\phi_{2}-\phi_{3}\right)}
\end{array},\right.
$$

where $\theta_{i}, i=1,2,3$, is the kicking strength for $i$ th dipole corrector. $\beta_{i}$ is the betatron amplitude function, and $\phi_{i}$ is the horizontal phase advance from the optics starting point.

Previous measurements of RHIC injection lattice optics indicate that injection arc optics are regular at the level of 5-10\%. Closure of bumps constructed using the above equations with model beta function and tunes also confirm this regularity. With constant bump amplitude, tune shifts from each of the 11 center bumps should match, and should match comparable patterns produced in the other five arcs. Outliers immediately indicate isolated problem sextupoles. Systematic sextupole wiring errors can be found by comparing measurements to a model - tune shifts from all bumps are compared to a model that includes sextupole feed-down, and potential errors can be simulated by reversing or zeroing selected sextupoles until the modified model matches the measurements. These simulations are detailed in the next section.

There are some tips for identifying wrong sextupole polarities in the middle 11 arc FODO horizontal bumps:

- If there are no wrong sextupole polarities, the tune shifts from bump to bump and the tune shift patterns from arc to arc should be similar. Wrong sextupole polarities can be easily identified from mismatched signs or amplitudes of tune shifts from bump to bump and/or from arc to arc.

- If one SF sextupole has a wrong polarity, it will only affect the tune shifts of the bump which covers this SF sextupole since one SF is only covered once by one bump. If one SD sextupole has a wrong polarity, it will only affect the tune shifts of two adjacent bumps since one SD is only covered by two adjacent bumps.

- From the RHIC optics model, if there are no wrong sextupole polarities, horizontal and vertical tune shifts from each bump should be positive.

- If the horizontal tune shift from one bump is negative and the vertical tune shift from this bump is excessively negative, there is a possibility that the SF in this bump has a wrong polarity. The name of the suspicious SF can be easily identified since each bump only covers one SF.

- If the vertical tune shifts from two adjacent bumps are negative or close to zero and the horizontal tune shift from these bumps are excessively positive, there is a possibility that the SD covered by these two bumps has a wrong polarity.

\subsection{Numerical simulations}

Numerical simulations were carried out to verify the above corrector based method. Based on the Blue ring injection optics, we set the kicking strengths of first horizontal dipole correctors to be $\theta_{1}=2.0 \times 10^{-4} \mathrm{rad}$.

Fig. 3 shows the horizontal and vertical tune shifts without any wrong sextupole polarities for the $13^{*} 2$ bumps in the first two arcs. The tune shifts from the middle 11 bumps repeat very well among each other, and the tune shift pattern is clearly the same between the first and second arcs.

Fig. 4 shows the tune shifts from these bumps when an SF sextupole in the first arc and an SD sextupole in the second arc have reversed polarity in the model. With the above sign and amplitude rules for the tune shifts, it is quite straightforward to pick out the sextupoles with incorrect polarities from the tune shift data. 


\section{Beam experiment in RHIC}

\subsection{Bump Setup}

Tab. 1 lists the correctors, bumps, and covered sextupoles for all 78 bumps $\left(6^{*} 13\right)$ in the RHIC Blue ring.

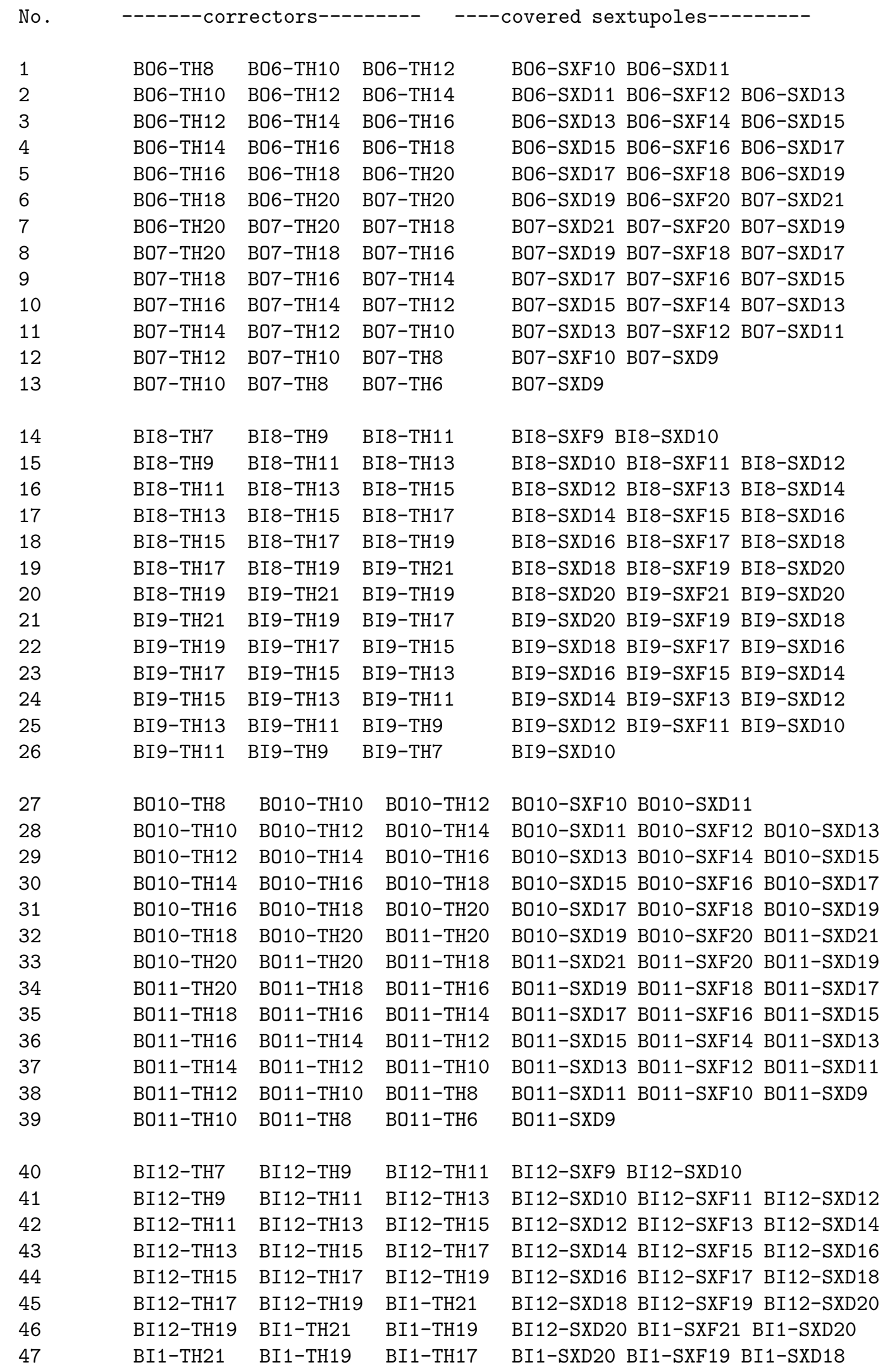




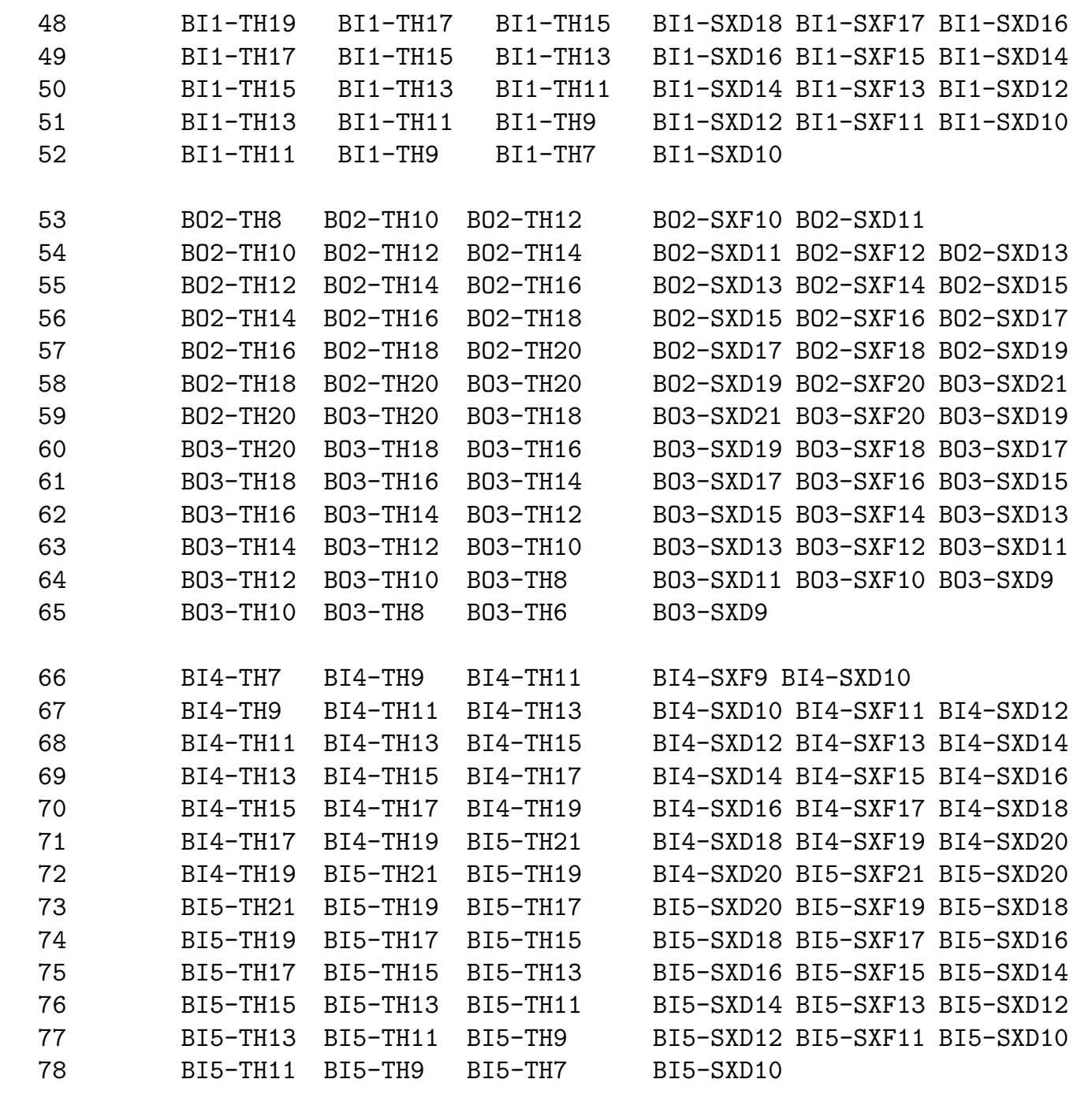

Tab. 2 lists -the correctors, bumps, and covered sextupoles for all 78 bumps (6*13) in -the RHIC Yellow ring.

\begin{tabular}{|c|c|c|c|c|}
\hline No. & \multicolumn{3}{|c|}{-------correctors---------- } & ----covered sextupoles--------- \\
\hline 1 & Y05-TH8 & Y05-TH10 & Y05-TH12 & Y05-SXF10 Y05-SXD11 \\
\hline 2 & Y05-TH10 & Y05-TH12 & Y05-TH14 & Y05-SXD11 Y05-SXF12 Y05-SXD13 \\
\hline 3 & Y05-TH12 & Y05-TH14 & Y05-TH16 & Y05-SXD13 Y05-SXF14 Y05-SXD15 \\
\hline 4 & Y05-TH14 & Y05-TH16 & Y05-TH18 & Y05-SXD15 Y05-SXF16 Y05-SXD17 \\
\hline 5 & Y05-TH16 & Y05-TH18 & YO5-TH2O & Y05-SXD17 Y05-SXF18 Y05-SXD19 \\
\hline 6 & Y05-TH18 & Y05-TH2O & YO4-TH2O & Y05-SXD19 Y05-SXF20 Y05-SXD21 \\
\hline 7 & Y05-TH2O & YO4-TH2O & Y04-TH18 & Y05-SXD21 YO4-SXF20 YO4-SXD19 \\
\hline 8 & YO4-TH2O & Y04-TH18 & Y04-TH16 & Y04-SXD19 Y04-SXF18 Y04-SXD17 \\
\hline 9 & Y04-TH18 & YO4-TH16 & YO4-TH14 & Y04-SXD17 Y04-SXF16 Y04-SXD15 \\
\hline 10 & Y04-TH16 & Y04-TH14 & Y04-TH12 & Y04-SXD15 Y04-SXF14 Y04-SXD13 \\
\hline 11 & Y04-TH14 & Y04-TH12 & Y04-TH10 & Y04-SXD13 Y04-SXF12 Y04-SXD11 \\
\hline 12 & Y04-TH12 & Y04-TH10 & Y04-TH8 & Y04-SXD11 Y04-SXF10 Y04-SXD9 \\
\hline 13 & Y04-TH10 & YO4-TH8 & YO4-TH6 & Y04-SXD9 \\
\hline 14 & YI3-TH7 & YI3-TH9 & YI3-TH11 & YI3-SXF9 YI3-SXD10 \\
\hline 15 & YІ3-ТH9 & YI3-TH11 & YI3-TH13 & YI3-SXD10 YI3-SXF11 YI3-SXD12 \\
\hline 16 & YI3-TH11 & YI3-TH13 & YI3-TH15 & YI3-SXD12 YI3-SXF13 YI3-SXD14 \\
\hline 17 & YI3-TH13 & YI3-TH15 & YI3-TH17 & YI3-SXD14 YI3-SXF15 YI3-SXD16 \\
\hline 18 & YI3-TH15 & YI3-TH17 & YI3-TH19 & YI3-SXD16 YI3-SXF17 YI3-SXD18 \\
\hline
\end{tabular}




\begin{tabular}{|c|c|c|c|c|}
\hline 19 & YI3-TH17 & YI3-TH19 & YI3-TH21 & YI3-SXD18 YI3-SXF19 YI3-SXD20 \\
\hline 20 & YI3-TH19 & YI3-TH21 & YI2-TH19 & YI3-SXD20 YI3-SXF21 YI2-SXD20 \\
\hline 21 & YI3-TH21 & YI2-TH19 & YI2-TH17 & YI2-SXD20 YI2-SXF19 YI2-SXD18 \\
\hline 22 & YI2-TH19 & YI2-TH17 & YI2-TH15 & YI2-SXD18 YI2-SXF17 YI2-SXD16 \\
\hline 23 & YI2-TH17 & YI2-TH15 & YI2-TH13 & YI2-SXD16 YI2-SXF15 YI2-SXD14 \\
\hline 24 & YI2-TH15 & YI2-TH13 & YI2-TH11 & YI2-SXD14 YI2-SXF13 YI2-SXD12 \\
\hline 25 & YI2-TH13 & YI2-TH11 & YI2-TH9 & YI2-SXD12 YI2-SXF11 YI2-SXD10 \\
\hline 26 & YI2-TH11 & YI2-TH9 & YI2-TH7 & YI2-SXD10 \\
\hline 27 & Y01-TH8 & Y01-TH10 & Y01-TH12 & Y01-SXF10 Y01-SXD11 \\
\hline 28 & Y01-TH10 & Y01-TH12 & Y01-TH14 & Y01-SXD11 Y01-SXF12 Y01-SXD13 \\
\hline 29 & Y01-TH12 & Y01-TH14 & Y01-TH16 & Y01-SXD13 Y01-SXF14 Y01-SXD15 \\
\hline 30 & Y01-TH14 & Y01-TH16 & Y01-TH18 & Y01-SXD15 Y01-SXF16 Y01-SXD17 \\
\hline 31 & Y01-TH16 & Y01-TH18 & Y01-TH20 & Y01-SXD17 Y01-SXF18 Y01-SXD19 \\
\hline 32 & Y01-TH18 & Y01-TH20 & Y012-TH20 & Y01-SXD19 Y01-SXF20 Y01-SXD21 \\
\hline 33 & YO1-TH20 & Y012-TH20 & Y012-TH18 & Y01-SXD21 Y012-SXF20 Y012-SXD19 \\
\hline 34 & Y012-TH20 & Y012-TH18 & 3 Y012-TH16 & Y012-SXD19 Y012-SXF18 Y012-SXD17 \\
\hline 35 & Y012-TH18 & Y012-TH16 & 6 Y012-TH14 & Y012-SXD17 Y012-SXF16 Y012-SXD15 \\
\hline 36 & Y012-TH16 & Y012-TH14 & 4 Y012-TH12 & Y012-SXD15 Y012-SXF14 Y012-SXD13 \\
\hline 37 & Y012-TH14 & Y012-TH12 & Y Y12-TH10 & Y012-SXD13 Y012-SXF12 Y012-SXD11 \\
\hline 38 & Y012-TH12 & Y012-TH10 & Y012-TH8 & Y012-SXD11 Y012-SXF10 Y012-SXD9 \\
\hline 39 & Y012-TH10 & Y012-TH8 & Y012-TH6 & Y012-SXD9 \\
\hline 40 & YI11-TH7 & YI11-TH9 & YI11-TH11 & YI11-SXF9 YI11-SXD10 \\
\hline 41 & YI11-TH9 & YI11-TH11 & YI11-TH13 & YI11-SXD10 YI11-SXF11 YI11-SXD12 \\
\hline 42 & YI11-TH11 & YI11-TH13 & 3 YI11-TH15 & YI11-SXD12 YI11-SXF13 YI11-SXD14 \\
\hline 43 & YI11-TH13 & YI11-TH15 & YI11-TH17 & YI11-SXD14 YI11-SXF15 YI11-SXD16 \\
\hline 44 & YI11-TH15 & YI11-TH17 & YI11-TH19 & YI11-SXD16 YI11-SXF17 YI11-SXD18 \\
\hline 45 & YI11-TH17 & YI11-TH19 & YI11-TH21 & YI11-SXD18 YI11-SXF19 YI11-SXD20 \\
\hline 46 & YI11-TH19 & YI11-TH21 & YI10-TH19 & YI11-SXD20 YI11-SXF21 YI10-SXD20 \\
\hline 47 & YI11-TH21 & YI10-TH19 & YI10-TH17 & YI10-SXD20 YI10-SXF19 YI10-SXD18 \\
\hline 48 & YI10-TH19 & YI10-TH17 & YI10-TH15 & YI10-SXD18 YI10-SXF17 YI10-SXD16 \\
\hline 49 & YI10-TH17 & YI10-TH15 & YI10-TH13 & YI10-SXD16 YI10-SXF15 YI10-SXD14 \\
\hline 50 & YI10-TH15 & YI10-TH13 & 3 YI10-TH11 & YI10-SXD14 YI10-SXF13 YI10-SXD12 \\
\hline 51 & YI10-TH13 & YI10-TH11 & YI10-TH9 & YI10-SXD12 YI10-SXF11 YI10-SXD10 \\
\hline 52 & YI10-TH11 & YI10-TH9 & YI10-TH7 & YI10-SXD10 \\
\hline
\end{tabular}

\begin{tabular}{|c|c|c|c|c|}
\hline 53 & Y09-TH8 & Y09-TH10 & Y09-TH12 & Y09-SXF10 Y09-SXD11 \\
\hline 54 & Y09-TH10 & Y09-TH12 & Y09-TH14 & Y09-SXD11 Y09-SXF12 Y09-SXD13 \\
\hline 55 & Y09-TH12 & Y09-TH14 & Y09-ТH16 & Y09-SXD13 Y09-SXF14 Y09-SXD15 \\
\hline 56 & Y09-TH14 & Y09-TH16 & Y09-TH18 & Y09-SXD15 Y09-SXF16 Y09-SXD17 \\
\hline 57 & Y09-TH16 & Y09-TH18 & YO9-TH20 & Y09-SXD17 Y09-SXF18 Y09-SXD19 \\
\hline 58 & Y09-TH18 & Y09-TH20 & Y08-TH20 & Y09-SXD19 Y09-SXF20 Y09-SXD21 \\
\hline 59 & YO9-TH2O & Y08-TH2O & Y08-TH18 & Y09-SXD21 Y08-SXF20 Y08-SXD19 \\
\hline 60 & YO8-TH2O & Y08-TH18 & Y08-TH16 & Y08-SXD19 Y08-SXF18 Y08-SXD17 \\
\hline 61 & Y08-TH18 & Y08-TH16 & Y08-TH14 & Y08-SXD17 Y08-SXF16 Y08-SXD15 \\
\hline 62 & Y08-TH16 & Y08-TH14 & Y08-TH12 & Y08-SXD15 Y08-SXF14 Y08-SXD13 \\
\hline 63 & YO8-TH14 & Y08-TH12 & Y08-TH10 & Y08-SXD13 Y08-SXF12 Y08-SXD11 \\
\hline 64 & Y08-TH12 & Y08-TH10 & Y08-TH8 & Y08-SXD11 Y08-SXF10 Y08-SXD9 \\
\hline 65 & Y08-TH10 & YO8-TH8 & YO8-TH6 & Y08-SXD9 \\
\hline 66 & YI7-TH7 & YI7-TH9 & YI7-TH11 & YI7-SXF9 YI7-SXD10 \\
\hline 67 & YI7-TH9 & YI7-TH11 & YI7-TH13 & YI7-SXD10 YI7-SXF11 YI7-SXD12 \\
\hline 68 & YI7-TH11 & YI7-TH13 & YI7-TH15 & YI7-SXD12 YI7-SXF13 YI7-SXD14 \\
\hline 69 & YI7-TH13 & YI7-TH15 & YI7-TH17 & YI7-SXD14 YI7-SXF15 YI7-SXD16 \\
\hline 70 & YI7-TH15 & YI7-TH17 & YI7-TH19 & YI7-SXD16 YI7-SXF17 YI7-SXD18 \\
\hline 71 & YI7-TH17 & YI7-TH19 & YI7-TH21 & YI7-SXD18 YI7-SXF19 YI7-SXD20 \\
\hline
\end{tabular}




$\begin{array}{llllll}72 & \text { YI7-TH19 } & \text { YI7-TH21 } & \text { YI6-TH19 } & \text { YI7-SXD20 YI7-SXF21 YI6-SXD20 } \\ 73 & \text { YI7-TH21 } & \text { YI6-TH19 } & \text { YI6-TH17 } & \text { YI6-SXD20 YI6-SXF19 YI6-SXD18 } \\ 74 & \text { YI6-TH19 } & \text { YI6-TH17 } & \text { YI6-TH15 } & \text { YI6-SXD18 YI6-SXF17 YI6-SXD16 } \\ 75 & \text { YI6-TH17 } & \text { YI6-TH15 } & \text { YI6-TH13 } & \text { YI6-SXD16 YI6-SXF15 YI6-SXD14 } \\ 76 & \text { YI6-TH15 } & \text { YI6-TH13 } & \text { YI6-TH11 } & \text { YI6-SXD14 YI6-SXF13 YI6-SXD12 } \\ 77 & \text { YI6-TH13 } & \text { YI6-TH11 } & \text { YI6-TH9 } & \text { YI6-SXD12 YI6-SXF11 YI6-SXD10 } \\ 78 & \text { YI6-TH11 } & \text { YI6-TH9 } & \text { YI6-TH7 } & \text { YI6-SXD10 }\end{array}$

These tables can be used to set up scripts to iterate through all the bumps, as described in the next section. They can also be used to correlate bump anomalies and possible miswired sextupoles during offline and online data analysis.

\subsection{Beam Experiment and Results}

In RHIC run_06, the above method was used to check all chromaticity sextupole polarities in RHIC during the APEX beam study period of March 22, 2006, starting at 14:50. The machine ramp and stone were pp30::injection. The rings were decoupled at injection, and the tunes were separated to avoid coupling interference in tune measurements. Scripts were written to adjust consecutive three-bumps with $+5 \mathrm{~mm}$ amplitudes through the arcs in each ring while monitoring and logging the tunes with the BBQ tunemeter system and orbits in all BPMs. Each bump took 25-30 seconds; the total data acquisition time for both rings was under two hours. The blue ring was scanned first, and then the yellow ring. The scripts record timestamps for each bump, and these records were later used to correlate bumps to logged BBQ tunemeter stripchart data.

Fig. 5 shows BBQ tunemeter data acquired during the scan of the blue ring. This data includes tests of the bumps, and proceeds in groups from arcs 10/11, 12/1,2/3,4/5,6/7, and 8/9 o'clock. There are two anomalies at 15:02 and 15:09 — these are the result of bumps that were not removed before the next bump was applied, and are not due to miswired sextupoles. Comparison of data in this figure to simulation indicates that all chromaticity sextupoles in the RHIC blue ring are wired correctly. A zoom of this data showing the details of tune response in correctly wired chromaticity sextupoles is shown in Fig. 6 .

Fig. 7 shows BBQ tunemeter data acquired during the scan of the yellow ring. This data also includes tests of the bumps, and proceeds in groups from arcs 10/11, 12/1, 2/3, 4/5,6/7, and 8/9 o'clock. This data is significantly harder to interpret from 16:08 to $16: 18$, when control system problems interfered with the three-bump script operation. This data was removed, and remaining yellow measurements were manually compared to the script logs and simulations. This comparison indicated that one sextupole, yo4-sxd9, had a reversed polarity. Once understood, this is fairly easy to see in data in Fig. 7 at 16:19:33, where the vertical tune clearly moves in the wrong direction. The wiring for this sextupole was corrected on April 4, 2006, and the correct polarity was confirmed with this method on April 12.

Both BBQ measurements show base tunes that gradually increase with time. This tune shift is about 0.002 up in both planes over about one hour, inconsistent with tune drift from persistent currents. This is likely due to some sort of systematic drift in the BBQ data, or feed-down effects of slow orbit drifts.

During the beam study, horizontal orbit motion due to in the arcs was also noted when bumps were applied. For constant frequency, the momentum shift due to a fractional closed orbit length change $\Delta C / C$ is $\delta=\gamma_{T}^{2} \Delta C / C$. Observed orbit motion outside of the bump in regular arcs of RHIC was $0.5 \mathrm{~mm}$, corresponding to a fractional energy change of $\delta=2.5 \times 10^{-4}$ and a fractional closed orbit length change of $\Delta C / C=5 \times 10^{-6}$. This is completely consistent with the $+5 \mathrm{~mm}$ horizontal bumps used along three-bumps that have $30 \mathrm{~m}$ between correctors in the RHIC arcs. The horizontal orbit motion from this effect in other chromaticity sextupoles is $10 \%$ of the bump size; this effect can be neglected when determining sextupole polarities, but should be included for measurement of optics from this data.

\section{Conclusions}

We have presented a beam-based method to check RHIC chromaticity sextupole polarities using local horizontal orbit three-bumps. This method was successfully used to check all sextupoles in RHIC in March, 2006. All blue chromaticity sextupoles had correct polarities verified; in the yellow ring, a single sextupole, yo4-sxd9, was found to be reversed. The wiring for this sextupole was corrected on April 42006 , and the corrected polarity was confirmed during another beam study on April 122006. 


\section{Acknowledgements}

We thank Johan Bengtsson, and Rama Calaga for help with the data acquisition during the beam experiment, and C-AD operations for their support. 


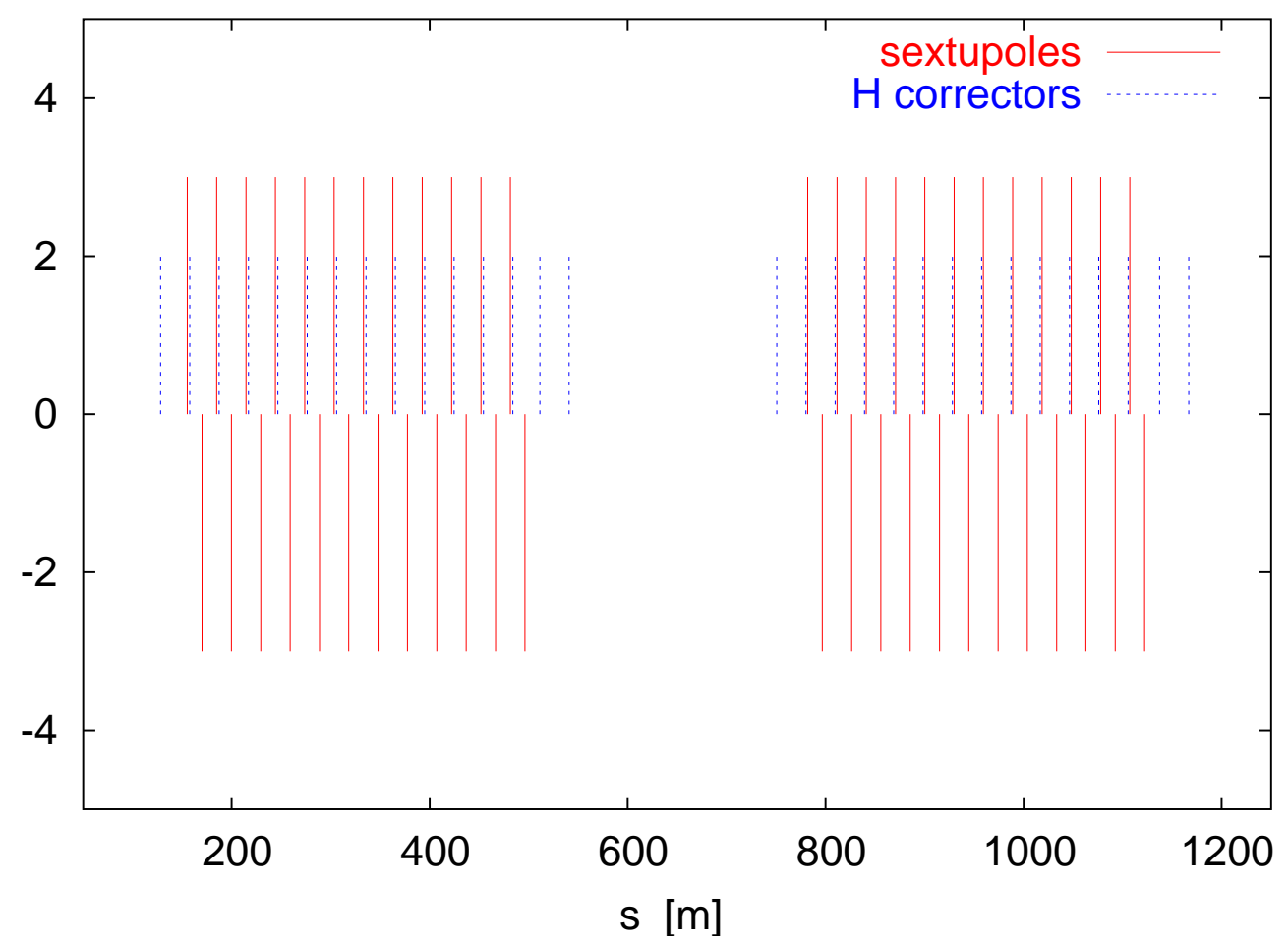

Figure 1: Sextupoles and horizontal correctors in the first two RHIC Blue ring arcs.

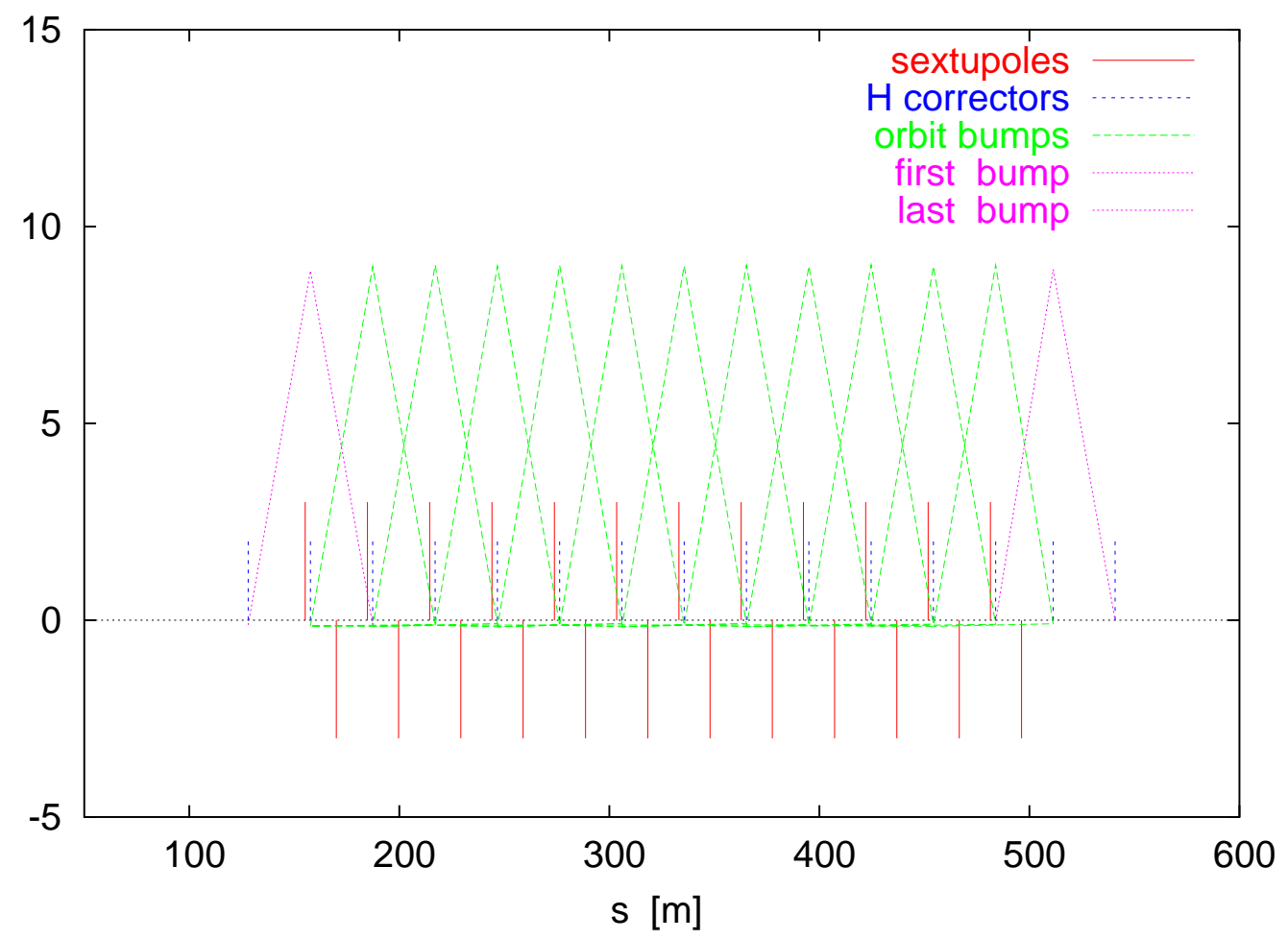

Figure 2: Proposed corrector based bumps in the first arc. 

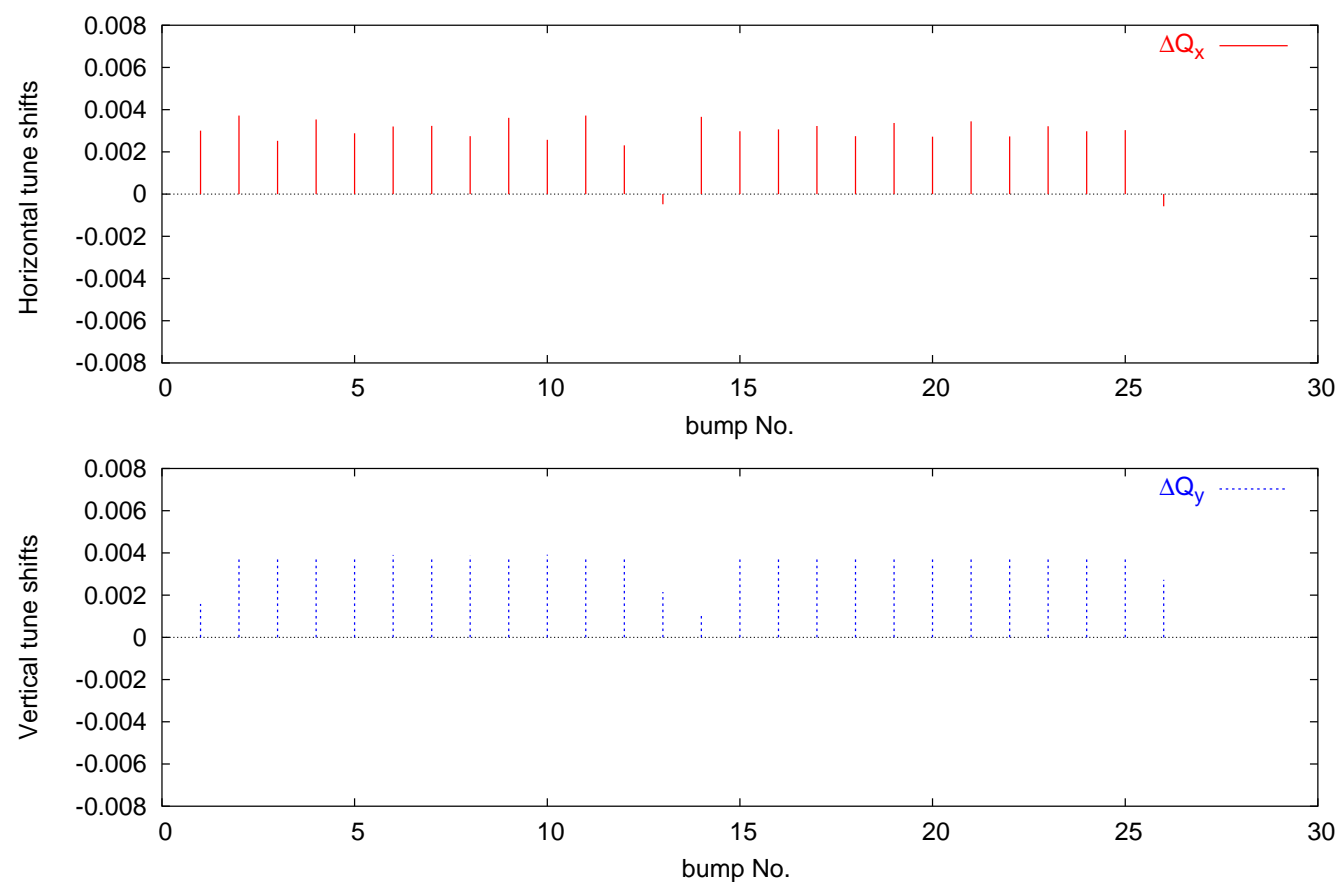

Figure 3: With no wrong sextupole polarities: simulated tune shifts for the bumps in the first two Blue ring $\operatorname{arcs}$
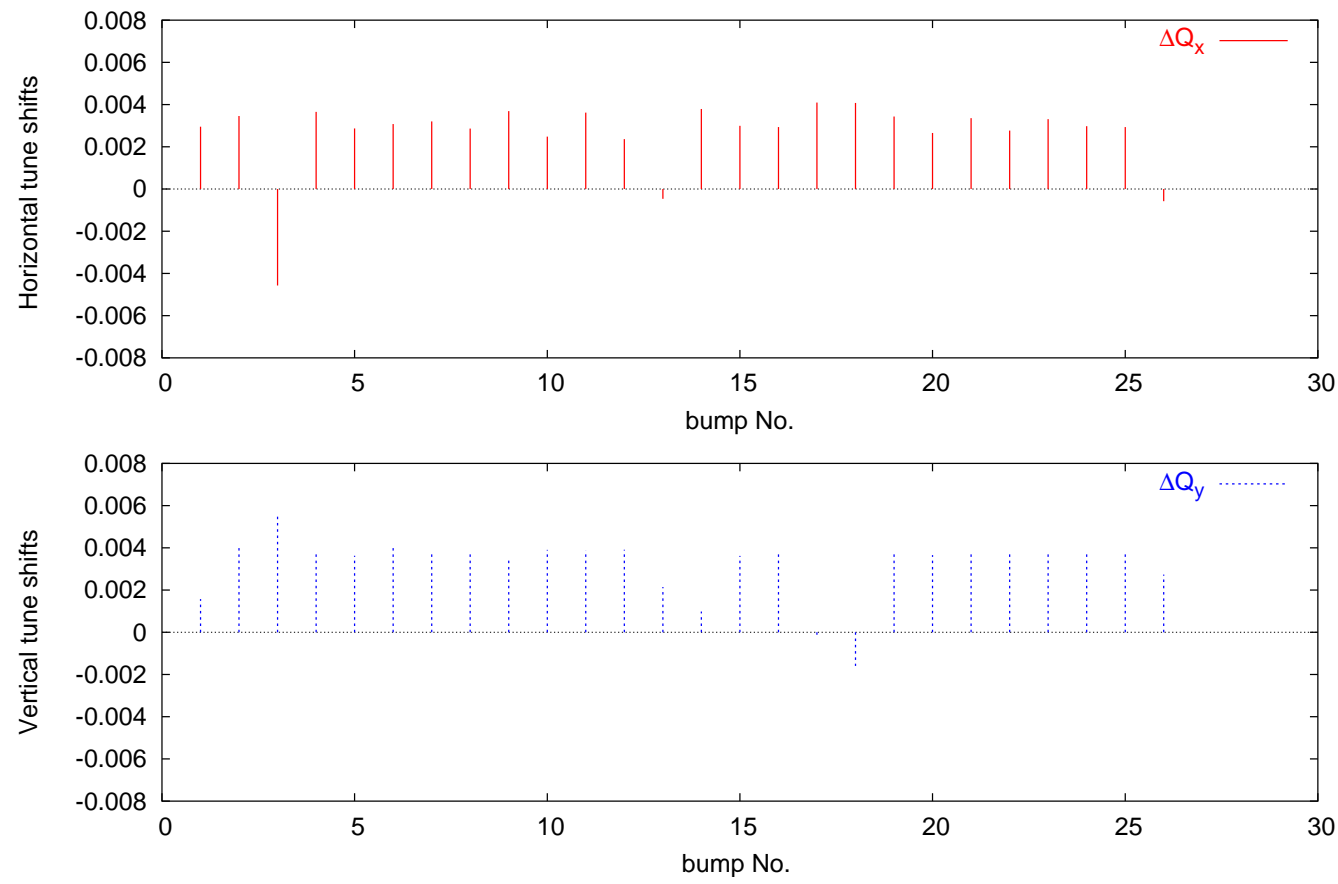

Figure 4: With two reversed sextupole polarities: simulated tune shifts for the bumps in the first two Blue ring arcs. 


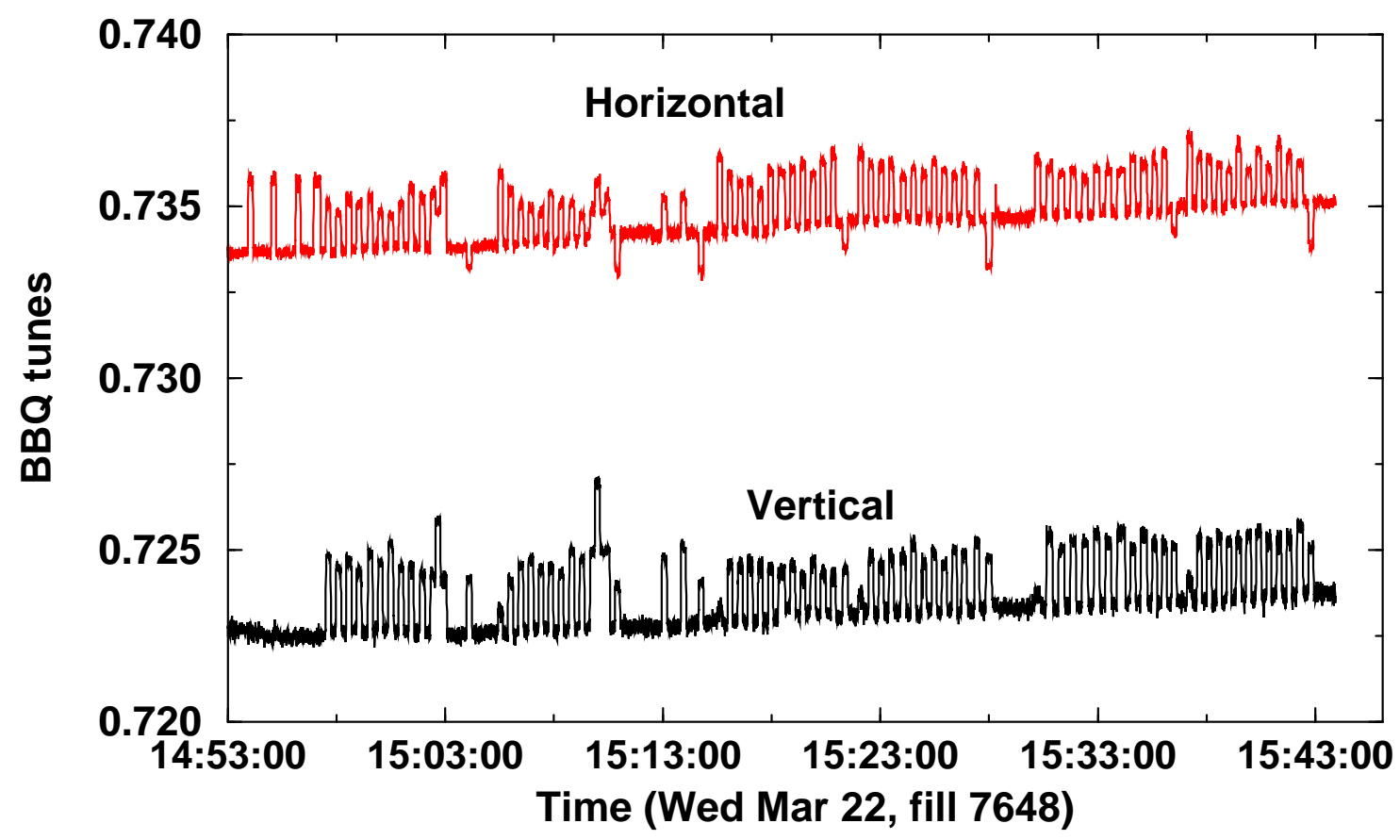

Figure 5: Blue BBQ tunemeter data acquired for scans of blue ring bumps in arcs 10/11, 12/1, 2/3, 4/5, 6/7, and 8/9 o'clock. Anomalies at 15:02 and 15:09 are the result of bumps not being removed, and are not due to miswired sextupoles.

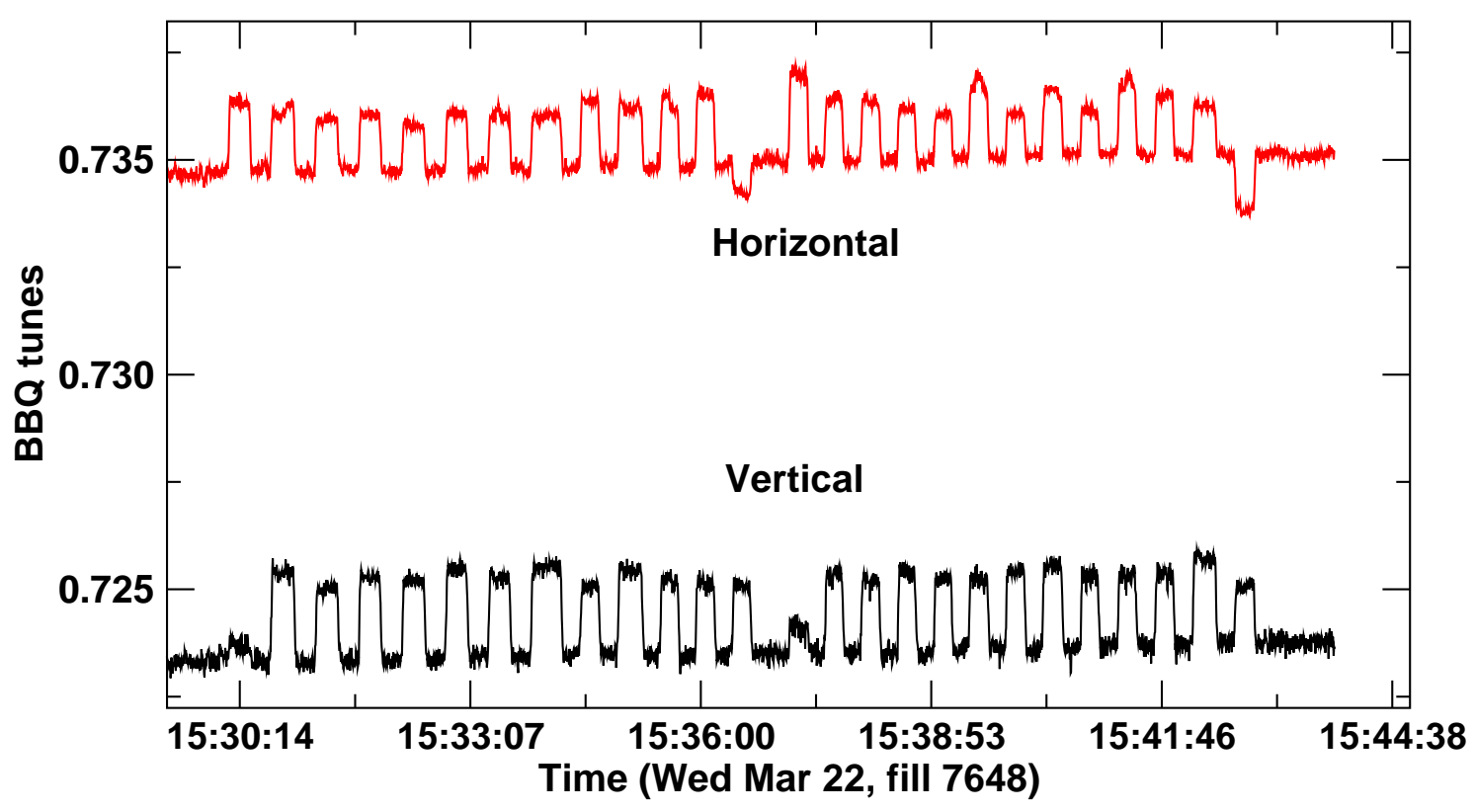

Figure 6: A zoom of the blue BBQ tunemeter data acquired for scans of blue ring bumps in $\operatorname{arcs} 6 / 7$, and $8 / 9$ o'clock, showing detailed bump configurations for correctly wired chromaticity sextupoles. 


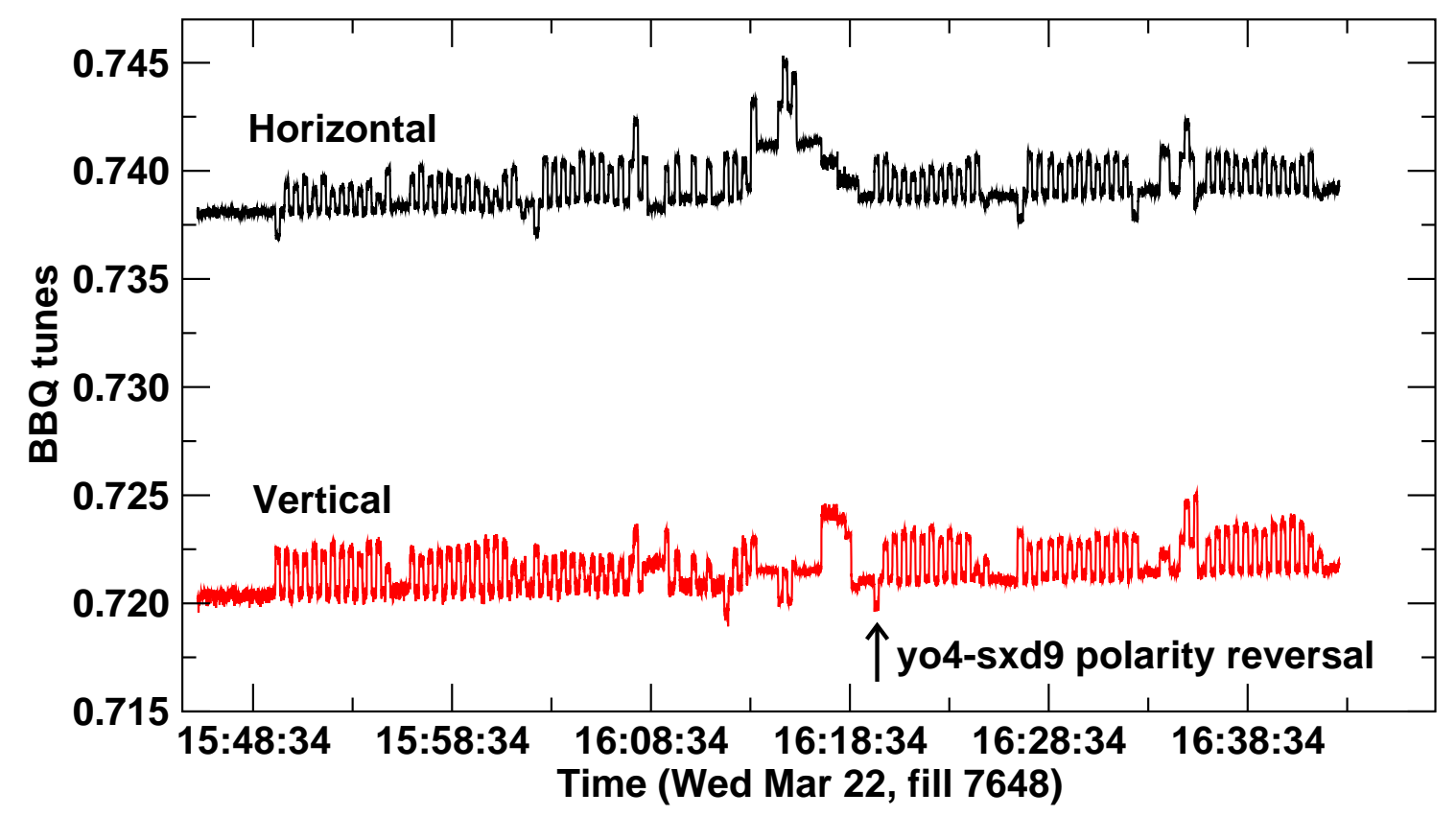

Figure 7: Yellow BBQ tunemeter data acquired for scans of yellow ring bumps in arcs 10/11, 12/1, 2/3, 4/5, 6/7, and 8/9 o'clock. The bump at 16:19 indicates a polarity reversal of chromaticity sextupole yo4-sxd9. 